\title{
Research on Urban Spatial Planning Strategy from the Perspective of Epidemic Prevention and Control
}

\author{
Yu SHI ${ }^{\mathrm{a}}$, Tao YANG ${ }^{\mathrm{a}, 1}$, Zongsheng $\mathrm{CHEN}^{\mathrm{b}}$, Tiemao SHI ${ }^{\mathrm{c}}$ and Zhenxing $\mathrm{LI}^{\mathrm{c}}$ \\ ${ }^{a}$ School of Design and Art, Shenyang Jianzhu University, Shenyang110168, China \\ ${ }^{\mathrm{b}}$ School of International College, Shenyang Jianzhu University, Shenyang110168, \\ China \\ ${ }^{\mathrm{c}}$ School of Architectural College, Shenyang University, Shenyang110044, China
}

\begin{abstract}
The global outbreak of COVID-19 has exposed the deficiency of urban space quality in terms of health and awareness of respiratory infectious diseases.This article analyzes the influencing factors of urban health mechanisms and simulates through infectious disease mechanisms and approaches. Finally Propose corresponding strategies and optimization methods for the optimization of urban space form and structure, and provide corresponding strategies and basis for healthy urban space planning.
\end{abstract}

Keywords. Epidemic prevention and control, urban space planning, health impact mechanism

\section{Introduction}

In the history, most viruses of epidemics invaded the respiratory system of the human body, which is exposed to nature and is the only one of the major circulatory systems of the human body that is directly connected and open to the outside world [1]. Epidemics are caused by infectious diseases, and there are three main ways of transmission: digestive system transmission, respiratory system transmission, and hematogenous transmission. Epidemics are regional, explosive and contagious [2]. Nowadays, with high social mobility and development, the epidemic that is most likely to spread on a large scale is the disease transmitted through the respiratory system, which is likely to become a regional epidemic and difficult to prevent and control. When invaded by viruses and other pathogenic microorganisms, the respiratory system is greatly challenged. Both air pollution and viruses will challenge our respiratory defenses. Facing frequent infectious diseases, planners should think carefully and deeply that how can they effectively prevent and control the epidemic through green space planning in highly densely populated cities, so as to reduce its threat to people's lives and make it less harmful to the economy and society.

\footnotetext{
${ }^{1}$ Tao Yang, School of Design and Art, Shenyang Jianzhu University, Shenyang110168, China; E-mail: 806009741@qq.com.
} 


\section{Influence Mechanisms Related to Urban Health}

Since "Healthy City" was put forward by WHO in the 1980s, there have been two directions in theoretical research: one is the impact of environmental factors on health, and the other is the research on healthy urban planning and design methods based on WHO's healthy city project [3].

\subsection{Impact of Environmental Factors on Health}

The research on the relationship between urban environment and public health is extensive, which can be divided into two aspects: health inhibition and promotion. In terms of health promotion, studies show that a well-designed environment can promote the physical activities of urban residents and have a positive impact on mental health [4]. Cervero R. Et al. believe that if the community combines higher development intensity with appropriate functional complexity, residents' life space will be relatively concentrated, and walking and cycling can be used to replace private cars and reduce carbon emissions [5]. Frumkin H. researches the role of recreation and sports facilities in promoting physical activity. He believes that about $50 \%$ of the population in the United States lacks regular exercise and leisure activities because of the lack of open activity space and recreation and sports facilities [6]. It is necessary to rationally allocate accessible open space and recreation and sports facilities, which can effectively encourage diversified physical activities. Mcintyre C.A. studies Canadian adults' walking, found that the more comfortable the environment is, the more enthusiastic and cohesive residents are, and physical activities will increase [7]. Health inhibition includes the lack of physical activity caused by unreasonable allocation of resources and respiratory diseases caused by automobile traffic exhaust. Pikora T. et al. found that the high density of road network around the residential space will lead to larger vehicle exhaust emissions, and residents will be more likely to be exposed to the polluted environment and respiratory diseases [8]. With medical statistics, Marshall.W.E. makes a correlation analysis on the influence of urban road network density and health status in plain cities [9]. The results show that with the decrease of street intersection density by $43.7 \%$ per square kilometer, the obesity, heart disease and hypertension incidence of a block population increases significantly, which also shows that low road network density is not conducive to physical activities dominated by walking, and incidence of chronic diseases such as obesity and cardiovascular disease will increase.

\subsection{Healthy Urban Planning and Design}

Green infrastructure is one of the effective ways to reduce urban particulate matter. There is a significant negative correlation between the green coverage rate and the particulate matter concentration in a block. Every $10 \%$ increase in the green coverage rate can lead to reduction of the PM10 and PM2.5 concentration by $13.83 \%$ and $7.58 \%$, respectively [10]. However, the research into plants' impact on abatement of particulate matter on the road space has always been controversial. The summary of previous studies shows that tall trees play a negative role in the street canyon [11], while low hedges can play a certain role in reducing the particulate matter concentration; on the open road space, vegetation plays a positive role in particulate matter reduction. The situation changes with the complex pollution reduction principle of plants [12]. The 
characteristics of vegetation, such as deposition rate, leaf area density, height, crown, configuration, leaf characteristics (hairy, waxy), seasonal variation and so on are related to particulate matter abatement.

Wang Lan et al quantitatively analyzed the correlation between respiratory system health (taking the prevalence rate of lung cancer as an index) and the spatial layout, scale and plant allocation of urban green space, and concluded that vegetation allocation is the main spatial element of green space significantly related to respiratory health (prevalence rate of lung cancer), and the influence of green space layout (such as average distance from patient's residence to park) and scale (such as public green space rate) and other factors is not obvious [13]. Shao Yuhan explained the influence and function between four street space types and urban street microclimate, and discussed the influence of landscape elements in urban street space on microclimate factors and those landscape elements' effectiveness [14]. Wang Lan and Zhao Xiaojing analyzed the potential relationship between particulate matter distribution, respiratory diseases and urban space environment, and put forward the theoretical framework and method path of healthy cities. Relevant follow-up research is also needed to better protect human health.

\section{Simulation Method of Infectious Diseases Pathogen Concentration}

\subsection{Characteristics and Pathogenic Mechanism of Infectious Diseases}

Usually, there are three ways of transmission of infectious diseases, namely, direct transmission, contact transmission and aerosol transmission. Direct transmission means that the droplets produced by patients when they talk and cough are inhaled by people close to them, resulting in infection. Contact transmission refers to that droplets containing viruses in the air after falling on the surface of an object are touched by people. Aerosol transmission refers to the infection caused by people inhaling the virus parasitic in the gas dispersion system formed on the surface of dust particles in the air. Novel coronavirus survived no more than 2 hours in a dry and ventilated environment, but aerosol spread indoors and outdoors has the characteristics of wide spread and long distance, which is likely to cause infection during this period of time. Therefore, what we focus on is how to make the virus lose its physiological activity in the urban space, or how to make the virus circulate through air circulation as soon as possible from the urban space with high population density.

\subsection{Basic Data of Spatial Simulation}

\subsubsection{Data Collection in the Space}

1) Weather Data in the Space. In the meteorological data testing phase, data of the sample space weather stations from China Meteorological Data Service Center are used as the initial boundary conditions to test the simulation space, including the prevailing wind direction throughout the year, the main wind direction in autumn and winter, and temperature and humidity conditions in the sample space, as shown in figure 1. 


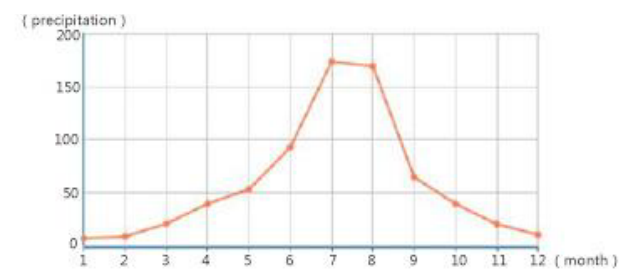

Figure 1. Monthy average precipitation over the years in Shenyang.

2) Block Data. The building combination mode in the block space has a great influence on the air flow. The scope of the sample area is identified by Google Map. The building outline in the space is vectorized in Arcgis, and the data of building enclosure mode, space enclosure, building height are extracted, as shown in figure 2.
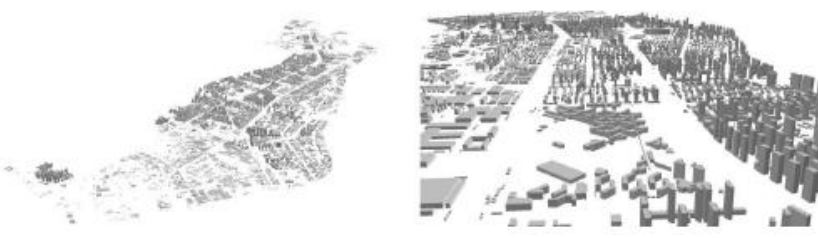

Figure 2. Shenyang Building Space Data of Humman New Area.

3) Vegetation Data. The main types of vegetation in the sample area were investigated, and the local tree species were collected as the main simulation object, and the model data such as leaf density and porosity of a single tree species were statistically analyzed, so that we can analyze the pollutant interception of different vegetation combinations next.

\subsubsection{Vegetation Allocation Model}

In order to focus on the effect of different plant allocation models on the interception of pathogenic pollutants, in the simulation of universal law, the plant allocation models are divided into: arbor shrub grass dominant type, shrub grass dominant type, tree shrub dominant type, shrub dominant type, arbor grass dominant type and grassland dominant type. Meanwhile, the combinations of each vegetation type are arranged and combined differently in space, coupling the law of seasonal change. The number of plants and plant spacing were repeatedly simulated as spatial variables, and the universal law of spatial allocation of local vegetation pollutant interception was obtained.

\subsubsection{Factor Correlation Analysis}

In order to obtain the universal law of pollutant interception mechanism in green space more accurately, the basic spatial data collected are sorted out, classified according to block data, vegetation data and meteorological data. Each subclass is taken as the variables of data analysis, correlation analysis is carried out in SPSS software, and the data type corresponding to the most coupled curve is selected as the reference conclusion of sample space simulation and optimization in the next step, as shown in figure 3 . 


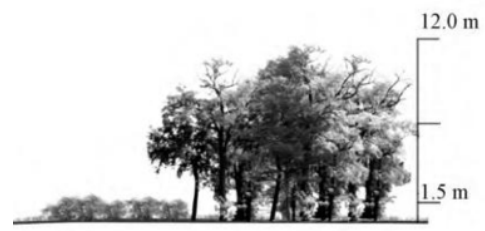

Figure 3. Spatial simulation of vegetation

\subsection{Pathogen Concentration Interception Mechanism}

By using ENVI-met to simulate the fine distribution characteristics of spatial pollution sources in the sample block and the interception of bioaerosol particles for different green space tree species, we can provide a scheme for the optimal selection of green space from the point of view of improving pathogenic pollutants, as shown in figure 4.
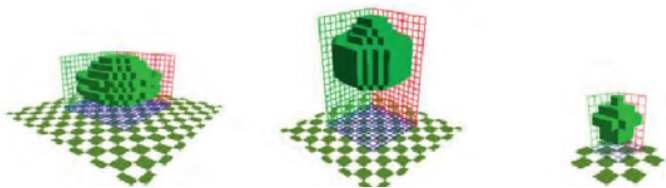

Figure 4. Simulation of Spatial form of Urban Green Space Based on GIS.

\subsection{Green Space Expression}

NDVI is a kind of green index based on visible light and near infrared spectrum surface reflectance. It can better describe the green coverage and evaluate the vegetation exposure in a specific area. NDVI is the most widely used vegetation index. At present, many studies use this index to describe green space situation. The calculation formula is:

$$
\mathrm{NDVI}=(\mathrm{NIR}-\mathrm{RED}) /(\mathrm{NIR}+\mathrm{RED})
$$

NIR refers to near-infrared spectral reflectance. RED refers to visible light reflectance. NDVI ranges from-1 to 1 . The higher the value, the higher the density of healthy vegetation, that is, the greenness is stronger. Negative value refers to water, snow and soil without vegetation.

Although NDVI is widely used as a tool for measuring green space exposure in epidemiological literature, it may overestimate publicly accessible green space by capturing all green areas. The public green space near the house has been shown to be related to the increased likelihood of physical use. In order to quantify publicly accessible green space, the GIS software ArcMap 10 is used to calculate the number of green space around participants' homes through manual exposure measurements. We use green space to categorize all parks, playgrounds, school playgrounds, cemeteries and other public open spaces, outline the characteristics of polygons to define the local green space, and estimate the green space area. We use Google to identify the green space area street view (also known as "ground truth"). Similar buffering analysis has previously been used to study green space and health outcomes. 


\subsection{Optimization of Spatial Structure of Urban Green Space at different Scales under the Background of Public Health Events}

At different scales, urban public green space with different spatial forms is simulated, and the results are compared horizontally. Circular radiating point green space, linear green space of corridor network and plane green space of green space patch are simulated respectively. The optimal solution of green space is obtained, and then the combination form of smaller scale green space under the most available space is simulated. The best space form under this scale is obtained. Finally, the optimization results are verified in reverse, and compared longitudinally, and a conclusion is drawn.

\section{Strategies and Optimization of Public Health Planning}

Our public health and epidemic prevention proves that many urban governance measures in China have played an important role and become a model of pandemic prevention and containment in the world, which has been imitated by some countries. These treatment measures are important methods for our country to quickly contain the pandemic and quickly return to work and production in a short period of time. First, it takes the basic units of community governance and community as the main body. Grass-roots communities play a key role in patient screening, medical treatment, whole-process follow-up, security of living resources, community psychological counseling and emotional assistance. The second is to establish and make full use of the urban big data management platform. In the early stage of the pandemic, the city big data was used to accurately predict the degree and scope of the spread of the pandemic, which provided decision-makers with the judgment of the development trend of the pandemic. Big data technology is used to sort out the life trajectory of infected people, track the contact history of people, locate the source of infection and close contact people, and provide methods to trace the source of the disease. Third, more consideration is given to the emergency needs of temporary construction. Planning and construction is always for permanent buildings or facilities, and we should make sure their sturdiness and durability in construction.

For cities with dense population and huge mobility, the focus of epidemic prevention and control is to quickly find and control the source of infection so that the disease does not produce new outbreak points; we should quickly and accurately grasp and control the route of transmission, and cut off the spread of the disease. We should control it within a limited range in order to reduce the damage and impact of the pandemic. We should quickly and accurately locate the susceptible population and adopt protective isolation measures to avoid the close contact between the susceptible population and the disease. The core of these pandemic prevention principles and concepts is to limit the mobility of cities. In fact, even during the period of high-risk pandemic prevention, the mobility of the city needs to be guaranteed to ensure that relief materials, personnel and equipment can enter quickly, and it is also necessary to provide basic living security to the public. These requirements for urban pandemic prevention are reflected differently on different scales of the city, and the different requirements are interrelated to form an overall pandemic prevention system.

1) Architectural level. Building is the direct space for citizens to live and work. In the urban management system, it is basically in a state of autonomy, but for the epidemic spread through air, contact, water, architecture is also the main place for the 
spread of infection. Therefore, architecture should be designed to meet the needs of disease prevention and control. We should carry out scientific and reasonable design in ventilation, wastewater treatment, public space and other aspects, and orderly selforganized and self-managed during the outbreak of the epidemic.

2) Community level. Community is not only the basic unit of urban administration, but also the specific place where the epidemic occurs, the place of transmission and the focus of prevention and control. The community needs to implement the prevention and control measures formulated by the city, and at the same time, quickly find and control the source of infection in the community. If the epidemic occurs, we also need to cut off the transmission routes between the community and the outside world, and limit the spread of the epidemic.

3) Urban level. This level is related to evaluation of, formulation of prevention and control measures for the overall epidemic situation in the city. On the basis of macroscopically grasping the data of the development of the epidemic situation and the speed of transmission, a model of urban spread of infectious diseases was constructed to predict and evaluate the scope and intensity of the epidemic.

These three levels support and cooperate with each other to form an organic system of urban compulsory epidemic prevention control and self-organizing epidemic prevention management, which can quickly and effectively contain the transmission and spread of the virus. The accurate grasp of epidemic information at the urban level comes from the accurate reporting of the community. The prevention and control measures at the urban level also depend on the specific implementation at the community level. These measures will be transmitted to the specific architectural level to ultimately provide support and related strategies for healthy urban planning.

\section{Conclusion}

The prevention and control of the epidemic can quickly and effectively prevent the spread of the virus through the organic combination of urban compulsory epidemic prevention and control and self-organizing epidemic prevention and control. City level of epidemic information accurately, accurately reported from the community, and the prevention and control measures on the level of city, also depends on the specific implementation at the community level, and conduction to the concrete construction level to provide support for healthy urban planning, so only coordinated from three different levels to understand the evolution of the epidemic to do to be effective and to provide basis for prevention and control of strategy.

\section{Acknowledgements}

Funding for this paper was provided by the National Natural Science Foundation of China (No.52008267).

\section{References}

[1] Friedenreich CM, Neilson HK, Lynch BM. State of the Epidemiological evidence on physical activity and cancer prevention. European Journal of Cancer. 2010 Sept; 46(14): 2593-2604. 
[2] Berg MD, Wendel-Vos W, et al. Health benefits of green spaces in the living environment: A systematic review of epidemiological studies. Urban Forestry \& Urban Greening. 2015 Aug; 14(4): 806-816.

[3] Li Q, Morimoto K, Kobayashi M, Inagaki H, Katsumata M, Hirata Y, Krensky A M. Visiting a forest, but not a city, increases human natural killer activity and expression of anti-cancer proteins. International. Journal of Immunopathology and Pharmacology. 2008 Jan; 21(1): 117-127.

[4] Richardson EA, Mitchell R, Hartig T, et al. Green cities and health: a question of scale? Journal of Epidemiology \& Community Health. 2012 Feb; 66(2): 160-165.

[5] Bixby H, Hodgson S, Fortunato L, et al. Associations between green space and health in English cities: An ecological, cross-sectional study. Plos One. 2015 Mar; 10(3): e0119495.

[6] Morimoto K, et al. Lifestyles and mental health status are associated with natural killer cell and lymphokine-activated killer cell activities. Science of The Total Environment. 2001 Apr; 270: 3-11.

[7] Hartig T, Evans GW, Jamner LD, Davis DS \& Garling T. Tracking restoration in natural and urban field settings. Journal of Environmental Psychology. 2003 Jun; 23(2): 109-123.

[8] Roe J \& Aspinall P. The restorative benefits of walking in urban and rural settings in adults with good and poor mental health. Health \& Place. 2011 Jan; 17(1): 103-113.

[9] Barton J \& Pretty J. What is the best dose of nature and green exercise for improving mental health? A multi-study analysis. Environmental Science \& Technology. 2010 Mar; 44(10): 3947-3955.

[10] Alcock I, White MP, Wheeler BW, Fleming LE and Depledge MH. Longitudinal effects on mental health of moving to greener and less green urban areas. Environmental Science \& Technology. 2014 Dec; 48(2): 1247-1255.

[11] Pope CA, Burnett RT, Thun MJ, et al. Lung cancer, cardiopulmonary mortality, and long-term exposure to fine particulate air pollution. JAMA. 2002 Mar; 287(9): 1132.

[12] Raaschou-Nielsen O, Andersen ZJ, Beelen R, et al. Air pollution and lung cancer incidence in 17 European cohorts: prospective analyses from the European study of cohorts for air pollution effects(ESCAPE). Lancet Oncology. 2013 Jul; 14(9): 813-822.

[13] Qian H, Li YG, Nielsen PV, et al. Spatial distribution of infection risk of sars transmission in a hospital ward. Build Environ. 2009 Aug; 44: 1651-1658.

[14] Wang L, Liao ShW, Zhao XJ. Exploration of approaches and factors of healthy city planning. Urban Planning International. 2016 Mar; 31(4): 4-9. 